\title{
Influence of the ICFF Decoupling Technique on the Stability of the Current Control Loop of a Grid-Tied VSC
}

\author{
Leonardo Marin, Andres Tarrasó, Ignacio Candela \\ Technical University of Catalonia \\ Terrassa, Barcelona, Spain \\ Email: leonardo.marin@upc.edu
}

\author{
Rebecca Rye \\ Virginia Polytechnic Institute and State University \\ Blacksburg, Virginia, United States of America \\ Email: beccarye@vt.edu
}

\author{
Pedro Rodriguez \\ Universidad de Loyola \\ Sevilla, Spain \\ Email: prodriguez@uloyola.es
}

\begin{abstract}
The control scheme of grid-tied converters is often implemented in the $d q$-frame due to simplicity of design. However, with this transformation, there exists an inherent cross-coupling term between the $d$ and $q$-channels which is often compensated for by using a feed-forward term in the current-control loop. It is shown, by applying the generalized Nyquist criterion (GNC) to the $d q$-frame ac impedance of the converter, that the inclusion of this decoupling term, in fact, degrades the stability of the controller when increasing the bandwidth of the synchronous reference frame phase-locked loop (SRF-PLL). Harware-in-the-loop (HIL) experiments are also conducted and verify these results.
\end{abstract}

Index Terms - small-signal, state-space, eigenvalues, impedance-based, generalized Nyquist criterion, inductor current feed-forward.

\section{INTRODUCTION}

In the last few decades, economic and environmental considerations have driven a significant growth in the use of renewable energies, mainly wind and photovoltaic, and this trend is expected to increase in the coming years [1]. However, the use of this type of energy is generally only possible by means of an adequate power-electronic-based interface, such as Voltage Source Converters (VSCs), which enables the control of variables such as voltage and frequency.

In both stand-alone and grid-connected mode, current control plays a key role in the operation of the VSC. Due its ease of implementation and satisfactory performance [2], one of the most commonly used approaches for current regulation is its implementation in a rotating Synchronous Reference frame (SRF), or $d q$ frame, by using Proportional-Integral (PI) controllers for the $d$ - and $q$-channels.

However, as is well known [3], there exists cross-coupling between the $d$ - and $q$-channels which produces a power quality degradation when current control is implemented in the SRF. Consequently, several decoupling techniques have been proposed to reduce the cross-coupling and improve the current transient performance [2-4]. Among the decoupling techniques, the Inductor Current Feed-Forward (ICFF) is widely used [3].

For grid-connected inverters, an algorithm for detecting the grid phase-angle is required for the synchronization [5]. Although a wide variety of synchronization schemes have been proposed, the Synchronous Reference Frame Phase-Locked Loop (SRF-PLL) is still widely used with single- and three-phase VSCs [6].

However, it is known that the synchronization loop introduces certain dynamics which negatively affect its stability, and this effect is strongly related to parameters such as the Short Circuit Ratio (SCR), the delay in the current control loop due the times of computation and Pulse-Width Modulation (PWM), the PLL bandwidth, and among others.

In this sense, the aim of this paper is to show that the use of the ICFF technique for decoupling the $d$ - and $q$-axis of the current control causes a negative effect on the stability of the system, and that this effect is more noticeable as the SRF-PLL bandwidth increases. This fact is proven by the application of two widely used approaches in small-signal stability analysis of VSC-based systems, such as the state-space-based approach [7] and the recently proposed impedance-based approach $[8,9]$. On one hand, the use of state space enables a direct comparison of the system's eigenvalues for the two cases: with and without ICFF. On the other hand, the impedance-based method is more intuitive and enables a better physical interpretation of the phenomenon.

One method to study the controller's small-signal stability as parameters are changed is to apply the generalized Nyquist criterion (GNC) for three-phase ac power systems, which uses the $d q$-frame impedance of the system under study [10]. 


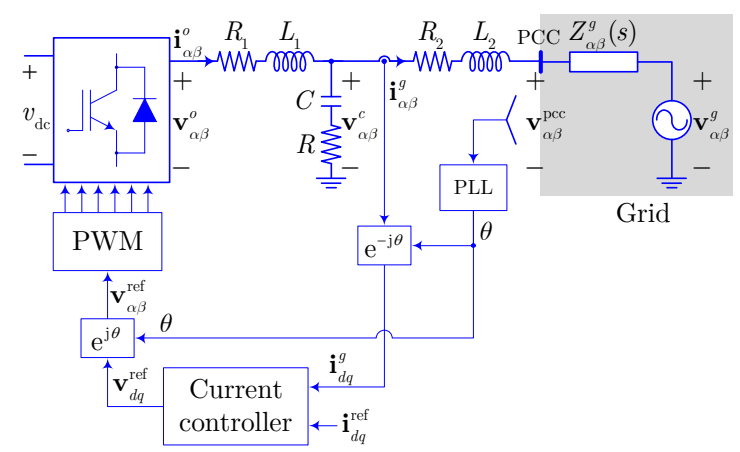

Fig. 1: One-line diagram of the system.

The remainder of the paper is stuctured as follows: section II describes the topology of the system under study. Section III derives the state-space model of the system to be used in section IV to analyze the effect of parametric changes on the stability of the system. Section V derives the impedance-based model of the system to which GNC is applied in section VI to analyze the small-signal stability under parametric changes. Section VII covers the experimental results using hardware-in-the-loop. Finally, section VIII is the conclusion.

\section{System Overview}

The system under study comprises a current control loop of a two-level three-phase VSC connected to the grid at the point of common coupling (PCC) through an LCL-filter. The simplified one-line diagram is shown in Fig. 1.

In this system, only balanced three-phase conditions have been assumed, i.e., voltages and currents contain only positive sequences. Therefore, the three-phase system has been reduced to an equivalent two-phase system expressed in the $\alpha \beta$ or $d q$-frame. In addition, the dc bus voltage, $v_{\mathrm{dc}}$, has been assumed as constant and the dynamics introduced by the switching scheme are disregarded.

In this paper, boldface letters are used to denote complex space vectors and complex transfer functions [11]. On the other hand, vectors referred to the stationary $\alpha \beta$ and $d q$-frame are denoted with the subscripts $\alpha \beta$ and $d q$ respectively, i.e., $\mathbf{x}_{\alpha \beta}=x_{\alpha}+\mathrm{j} x_{\beta}$ and $\mathbf{x}_{d q}=x_{d}+\mathrm{j} x_{q}$.

Fig. 2 shows a non-linear block diagram of the current controller. The PLL provides the phase angle needed for the transformation of signals from $\alpha \beta$ to $d q$ and vice versa.

The current controller is implemented in the $d q$-frame and consists of a PI controller, whose transfer function is denoted by $G_{c}(s)$, together with the ICFF, $j \omega_{1} L$, which is used to decouple the $d q$ axes cross-coupling [2], and whose effect on the system stability is what this paper is intended to analyse. Theoretically, to achieve a total decoupling, $L$ should include the value of the grid inductance. However, since this value is generally unknown and strongly changing according of the grid-operation

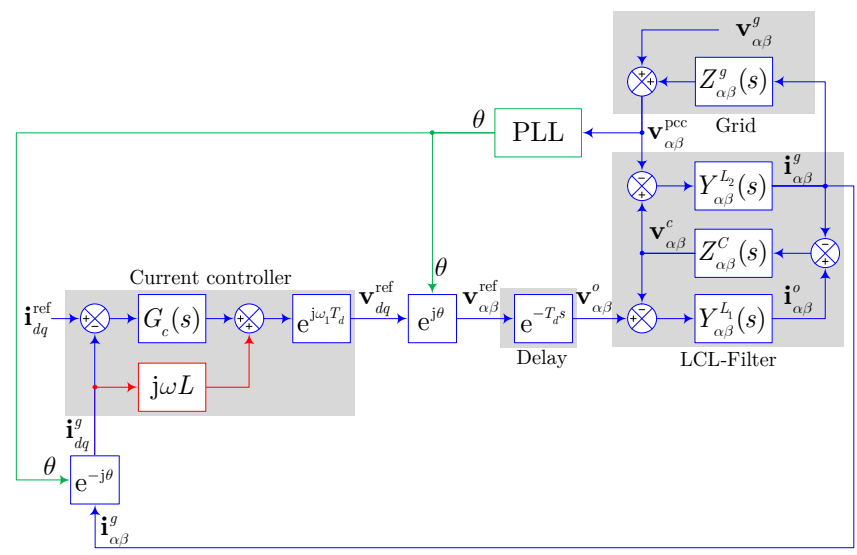

Fig. 2: Block diagram of the non-linear system.

conditions, it is assume that $L$ includes at least the total inductance of the LCL-filter, i.e., $L=L_{1}+L_{2}$.

The digital implementation of the current control introduces a delay, $\mathrm{e}^{-s T_{d}}$, due to the computation time and PWM scheme. It is common to assume that the time delay $T_{d}$ is $1.5 T_{s}$ [13], where $T_{s}$ is the sampling time of the current control. In the current controller, $\mathrm{e}^{\mathrm{j} \omega_{1} T_{d}}$ is a correction factor for the small angular displacement resulting from the transformation of the delay from $\alpha \beta$ to $d q[14]$.

A passively-damped LCL-filter, as shown in Fig. 1, has been assumed. The converter-side and the grid-side inductors are represented by $L_{1}$ and $L_{2}$, respectively, while $C$ is the shunt capacitor; $R_{1}$ and $R_{2}$ refer to the internal resistance of $L_{1}$ and $L_{2}$, respectively, and $R$ is the damping resistor in the shunt branch. The converter output voltage is given by $\mathbf{v}_{\alpha \beta}^{o}$ while $\mathbf{v}_{\alpha \beta}^{\mathrm{pcc}}$ denotes the $\mathrm{PCC}$ voltage. A functional block diagram of the LCL-filter is shown Fig. 2 where

$$
\begin{aligned}
Y_{\alpha \beta}^{L_{1}}(s) & =\frac{1}{L_{1} s+R_{1}} \\
Y_{\alpha \beta}^{L_{2}}(s) & =\frac{1}{L_{2} s+R_{2}} \\
Z_{\alpha \beta}^{C}(s) & =\frac{1}{C s}+R
\end{aligned}
$$

\section{State-SPACE MOdEL OF The System}

The derivation procedure to obtain the non-linear state-space model of the system under study is detailed in [15]. Then, in order to apply linear analysis techniques based on eigenvalues, this model is linearised around a operation point, yielding a 12th-order small-signal state-space model which can be expressed in the classical form

$$
\frac{\mathrm{d} \mathbf{x}}{\mathrm{d} t}=\mathbf{A} \mathbf{x}+\mathbf{B u}
$$




\begin{tabular}{lccc}
\hline \multirow{2}{*}{ Parameter } & \multirow{2}{*}{ Symbol } & \multicolumn{2}{c}{ VSC } \\
\cline { 3 - 4 } & & $100 \mathrm{kVA}$ & $4 \mathrm{MVA}$ \\
\hline Nominal ac voltage & $V_{n}$ & $400 \mathrm{~V}$ & $690 \mathrm{~V}$ \\
dc bus voltage & $v_{d c}$ & $750 \mathrm{~V}$ & $1150 \mathrm{~V}$ \\
Switching frequency & $f_{s w}$ & $3150 \mathrm{~Hz}$ & $2000 \mathrm{~Hz}$ \\
Sampling frequency & $f_{s}$ & $6300 \mathrm{~Hz}$ & $4000 \mathrm{~Hz}$ \\
\hline & $L_{1}$ & $0.1909 \mathrm{pu}$ & $0.1716 \mathrm{pu}$ \\
& $R_{1}$ & $0.0057 \mathrm{pu}$ & $0 \mathrm{pu}$ \\
LCL-filter & $L_{2}$ & $0.0987 \mathrm{pu}$ & $0.0581 \mathrm{pu}$ \\
& $R_{2}$ & $0.0016 \mathrm{pu}$ & $0 \mathrm{pu}$ \\
& $C$ & $0.0265 \mathrm{pu}$ & $0.0374 \mathrm{pu}$ \\
& $R$ & $0.3906 \mathrm{pu}$ & $0.1470 \mathrm{pu}$ \\
\hline \multirow{2}{*}{ Curren controller } & $k_{p}$ & $1.7578 \mathrm{pu}$ & $1.2602 \mathrm{pu}$ \\
& $k_{i}$ & $78.1250 \mathrm{pu} / \mathrm{s}$ & $63.0120 \mathrm{pu} / \mathrm{s}$ \\
\hline Time delay & $T_{d}$ & $0.2381 \mathrm{~ms}$ & $0.3750 \mathrm{~ms}$ \\
\hline Short circuit ratio & $\mathrm{SCR}$ & 10 & 10 \\
\hline
\end{tabular}

TABLE I: Parameters of the VSCs.

where $\mathbf{x}$ and $\mathbf{u}$ are given by

$$
\begin{gathered}
\mathbf{x}=\left[\begin{array}{lllllllll}
\theta & x^{\mathrm{pll}} & x_{d}^{\mathrm{cc}} & x_{q}^{\mathrm{cc}} & x_{d}^{d} & x_{q}^{d} & \cdots \\
& \cdots & i_{d}^{o} & i_{q}^{o} & i_{d}^{g} & i_{q}^{g} & v_{d}^{c} & v_{q}^{c}
\end{array}\right]^{T} \\
\mathbf{u}=\left[\begin{array}{lllll}
i_{d}^{\mathrm{ref}} & i_{q}^{\mathrm{ref}} & v_{d}^{g} & v_{q}^{g}
\end{array}\right]^{T}
\end{gathered}
$$

\section{Effect of Parametric Changes}

One of the main design parameters of the SRF-PLL is its bandwidth, $f_{\mathrm{bw}}^{\text {pll }}$, which will affect the performance of the controller overall.

Fig. 3 shows the trajectories of the eigenvalues of the state-space model metioned in the previous section as the $f_{\mathrm{bw}}^{\text {pll }}$ changes for two power ratings: $100 \mathrm{kVA}$ and $4 \mathrm{MVA}$. The parameters for each case are shown in Table I. Black arrows denote the direction of increasing $f_{\mathrm{bw}}^{\text {pll while red }}$ and blue circles denote the operation of the VSC with and without ICFF, respectively.

The value of $f_{\mathrm{bw}}^{\mathrm{pll}}$ for which the system becomes unstable is indicated for all cases with red and blue arrows. In the case of the $100 \mathrm{kVA}$ VSC, the maximum stable PLL bandwidth achieved without ICFF $(200 \mathrm{~Hz})$ is about $30 \%$ greater than with ICFF $(155 \mathrm{~Hz})$. In the case of the 4 MVA VSC, the difference is more severe: the PLL bandwidth reached without ICFF $(265 \mathrm{~Hz})$ is about $130 \%$ greater than with ICFF $(115 \mathrm{~Hz})$.

\section{IMPEDANCE-BASED MODEL OF THE SYSTEM}

Although the linearised state-space model is a mature analysis tool and is widely used for the stability analysis of power systems, it does not provide a clear explanation on the difference in the stability margins for when the ICFF is used versus when it is not used. Therefore, in this section, an impedance-based model of the system, based on the work presented in [6], is developed in order to provide more insight on the phenomenon.

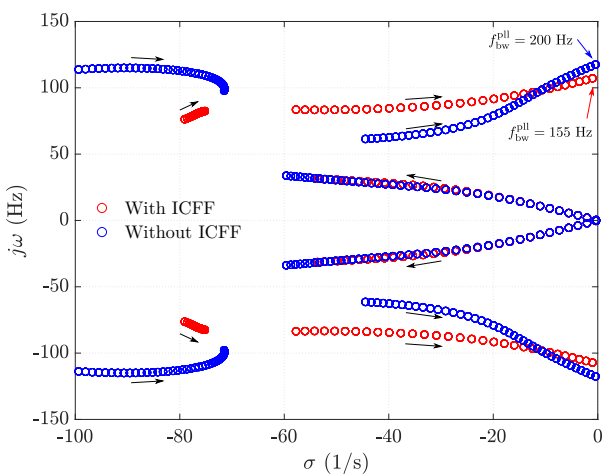

(a) $100 \mathrm{kVA}$

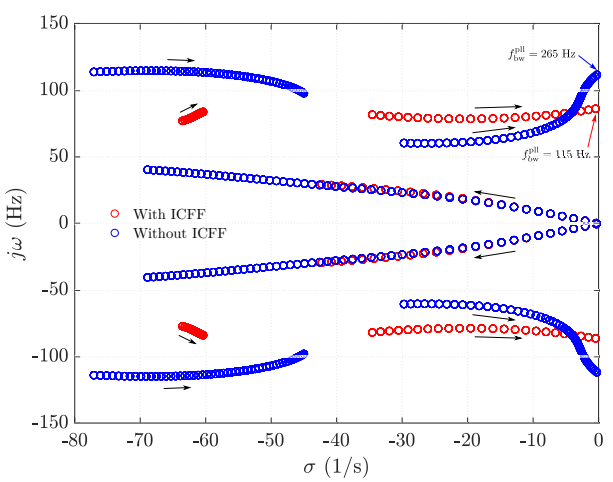

(b) 4 MVA.

Fig. 3: System Eigenvalues for a SRF-PLL bandwidth sweep: (a) $100 \mathrm{kVA}$ VSC, (b) 4 MVA VSC.

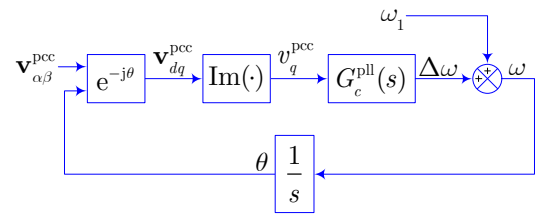

Fig. 4: Block diagram of the SRF-PLL.

The impedance-based model is developed in two steps: 1) linearising each blocks respectively, and 2) assembling these linearised building blocks by applying control-block reduction techniques.

\section{A. Synchronous reference frame phase-locked loop}

Fig. 4 depicts the block diagram of the SRF-PLL, where the Park transformation is used for phase detection and the output $q$-axis voltage is regulated by a PI controller, $G_{c}^{\mathrm{pll}}(s)=k_{p}^{\mathrm{pll}}+k_{i}^{\mathrm{pll}} / s$, for phase tracking [6].

The small-signal closed loop response the SRF-PLL is given by $[6]$

$$
\Delta \theta=G_{\mathrm{cl}}^{\mathrm{pll}}(s) \operatorname{Im}\left(\mathbf{v}_{d q}^{\mathrm{pcc}}\right)
$$

where

$$
G_{\mathrm{cl}}^{\mathrm{pll}}(s)=\frac{k_{p}^{\mathrm{pll}} s+k_{i}^{\mathrm{pll}}}{s^{2}+k_{p}^{\mathrm{pll}} s+k_{i}^{\mathrm{pll}}}
$$




\section{B. Effect of SRF-PLL on current control loop}

Because of the transformation of voltage and current signals from $\alpha \beta$ to $d q$ and vice versa, any perturbation coming from the grid will be transferred to the current control loop through the SRF-PLL as a perturbation in the phase-angle, $\theta$, as shown the green lines in Fig. 2.

Phase-angle disturbances have a twofold effect on the current control: on one hand, they will modify the measured current, $\mathbf{i}_{\alpha \beta}^{g}$, due the transformation to $d q$-frame; on the other hand, they will modify the voltage reference, $\mathbf{v}_{d q}^{\text {ref }}$, coming from the current control because of the transformation to $\alpha \beta$-frame.

Since phase-angle perturbations are fully transmitted to the measured current, they would exist a third path if ICFF is used, as shown by the red lines in Fig. 2. This possibility has not been taken in account [6].

1) Effect on the measured current: The measured current, $\mathbf{i}_{\alpha \beta}^{g}$, is transformed to the $d q$-frame using the Park transformation as follows:

$$
\mathbf{i}_{d q}^{g}=\mathrm{e}^{-\mathrm{j} \theta} \mathbf{i}_{\alpha \beta}^{g}
$$

In (7), $\mathbf{i}_{d q}^{\mathrm{ref}}$ is a function of $\mathbf{i}_{\alpha \beta}^{\mathrm{ref}}$ and $\theta$ and is linearised as follows:

$$
\begin{aligned}
\delta \mathbf{i}_{d q}^{g} & =\left.\frac{\partial \mathbf{i}_{d q}^{g}}{\partial \mathbf{i}_{\alpha \beta}^{g}}\right|_{0} \Delta \mathbf{i}_{\alpha \beta}^{g}+\left.\frac{\partial \mathbf{i}_{d q}^{g}}{\partial \theta}\right|_{0} \Delta \theta \\
& =\underbrace{\mathrm{e}^{-\mathbf{j} \theta_{0}} \Delta \mathbf{i}_{\alpha \beta}^{g}}_{\Delta \mathbf{i}_{d q}^{g}}-j \underbrace{\mathrm{je}^{-\mathrm{j} \theta_{0}} \mathbf{i}_{\alpha \beta 0}^{g}}_{\mathbf{i}_{d q 0}^{g}} \Delta \theta
\end{aligned}
$$

where the subscript " 0 " denotes the operating point of the linearisation. Eq. (8) shows that, in small-signal, the total current variation, $\delta \mathbf{i}_{d q}^{g}$, results from the linear combination of the current variation itself, $\Delta \mathbf{i}_{d q}^{g}$, and the phase-angle variation, $\Delta \theta$. Therefore,

$$
\delta \mathbf{i}_{d q}^{g}=\Delta \mathbf{i}_{d q}^{g}-\Delta \mathbf{i}_{d q}^{\text {pll }}
$$

where

$$
\Delta \mathbf{i}_{d q}^{\mathrm{pll}}=\mathbf{j} \mathbf{i}_{d q 0}^{g} \Delta \theta
$$

By replacing $\Delta \theta$ of (5) in (10)

$$
\Delta \mathbf{i}_{d q}^{\mathrm{pll}}=\mathbf{Y}_{d q}^{\mathrm{pll}}(s) \operatorname{Im}\left(\mathbf{v}_{d q}^{\mathrm{pll}}\right)
$$

where

$$
\mathbf{Y}_{d q}^{\mathrm{pll}}(s)=\mathrm{ji}_{d q 0}^{g} G_{\mathrm{cl}}^{\mathrm{pll}}(s)
$$

2) Effect on the voltage reference: As shown in Fig. 2 the voltage reference, $\mathbf{v}_{d q}^{\text {ref }}$, coming from the current control is transformed to $\alpha \beta$-frame by using the inverse Park transformation as follows:

$$
\mathbf{v}_{\alpha \beta}^{\mathrm{ref}}=\mathrm{e}^{\mathrm{j} \theta} \mathbf{v}_{d q}^{\mathrm{ref}}
$$

Proceeding as in (8), linearisation of (13) results in

$$
\delta \mathbf{v}_{d q}^{\mathrm{ref}}=\Delta \mathbf{v}_{d q}^{\mathrm{ref}}+\Delta \mathbf{v}_{d q}^{\mathrm{pll}}
$$

where

$$
\Delta \mathbf{v}_{d q}^{\mathrm{pll}}=\mathbf{G}_{d q}^{\mathrm{pll}}(s) \operatorname{Im}\left(\mathbf{v}_{d q}^{\mathrm{pll}}\right)
$$

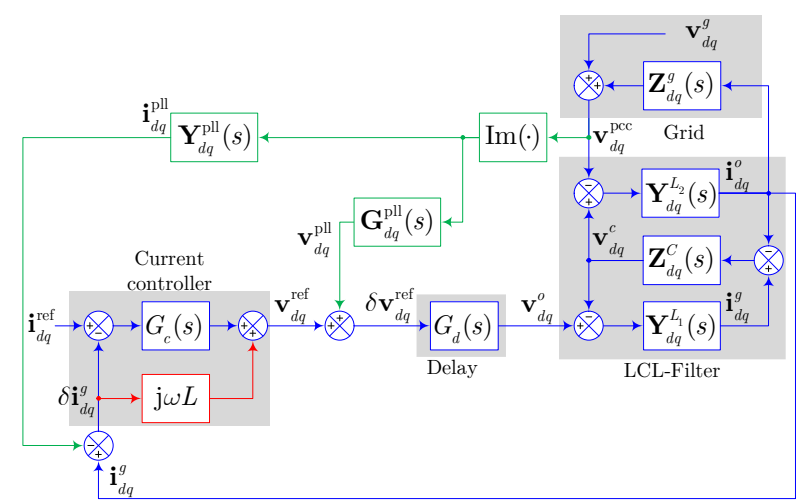

Fig. 5: Block diagram of the linearised system.

and

$$
\mathbf{G}_{d q}^{\mathrm{pll}}(s)=\mathrm{j} \mathbf{v}_{d q 0}^{\mathrm{ref}} G_{\mathrm{cl}}^{\mathrm{pll}}(s)
$$

\section{LCL-filter and grid}

The transformation of a relation $\mathbf{y}_{\alpha \beta}=G_{\alpha \beta}(s) \mathbf{u}_{\alpha \beta}$ gives a frequency translation of $s \rightarrow s+\mathrm{j} \omega_{1}$ when transformed to the $d q$-frame [11]. Therefore, the transfer functions of the LCL-filter and the grid can be transformed from the $\alpha \beta$ - to the $d q$-frame as follows:

$$
\begin{aligned}
\mathbf{Y}_{d q}^{L_{1}} & =Y_{\alpha \beta}^{L_{1}}\left(s+\mathrm{j} \omega_{1}\right) \\
\mathbf{Y}_{d q}^{L_{2}} & =Y_{\alpha \beta}^{L_{2}}\left(s+\mathrm{j} \omega_{1}\right) \\
\mathbf{Z}_{d q}^{C} & =Z_{\alpha \beta}^{C}\left(s+\mathrm{j} \omega_{1}\right) \\
\mathbf{Z}_{d q}^{g} & =Z_{\alpha \beta}^{g}\left(s+\mathrm{j} \omega_{1}\right)
\end{aligned}
$$

\section{Delay of the current control}

Due the frequency translation, the delay $\mathrm{e}^{-T_{d} s}$ in the $\alpha \beta$-frame is transformed such that $\mathrm{e}^{-T_{d}\left(s+\mathrm{j} \omega_{1}\right)}=$ $\mathrm{e}^{-\mathrm{j} \omega_{1} T_{d}} \mathrm{e}^{-T_{d} s}$. The factor $\mathrm{e}^{\mathrm{j} \omega_{1} T_{d}}$ of the current control cancels out the small angular displacement, $-\omega_{1} T_{d}$. If a first-order Padé approximation is used in order to rationalize the factor $\mathrm{e}^{-T_{d} s}$, the delay can be expressed as

$$
\mathrm{e}^{-T_{d} s} \approx G_{d}(s)=\frac{2-T_{d} s}{2+T_{d} s}
$$

Fig. 5 shows a block diagram of the small-signal model where the signals and the blocks are expressed in the $d q$-frame. By applying control-block reduction techniques, the small signal model of Fig. 5 can be equivalently depicted as shown in Fig. (5), where de admittances are given by

$$
\begin{aligned}
\mathbf{Y}_{d q}^{\mathrm{cl}} & =\frac{\mathbf{Y}_{d q}^{o}}{1+\left(G_{c}-\mathrm{j} \omega_{1} L\right) G_{d} \mathbf{Y}_{d q}^{p}} \\
\mathbf{Y}_{d q}^{\mathrm{pllt}} & =-\frac{G_{d} \mathbf{Y}_{d q}^{p}\left(G_{c} \mathbf{Y}_{d q}^{\mathrm{pll}}+\mathbf{G}_{d q}^{\mathrm{pll}}\right)}{1+\left(G_{c}-\mathrm{j} \omega_{1} L\right) G_{d} \mathbf{Y}_{d q}^{p}} \\
\mathbf{Y}_{d q}^{\mathrm{de}} & =\frac{\mathrm{j} \omega_{1} L G_{d} \mathbf{Y}_{d q}^{p} \mathbf{Y}_{d q}^{\mathrm{pll}}}{1+\left(G_{c}-\mathrm{j} \omega_{1} L\right) G_{d} \mathbf{Y}_{d q}^{p}}
\end{aligned}
$$




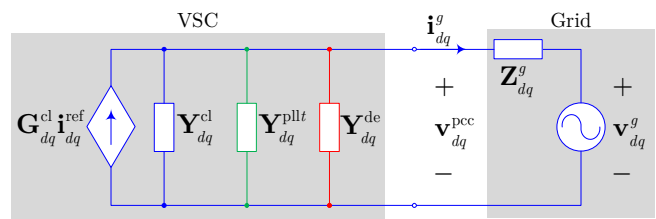

Fig. 6: Impedance-based model of the system.

where

$$
\begin{gathered}
\mathbf{Y}_{d q}^{p}=\frac{\mathbf{Y}_{d q}^{L_{1}} \mathbf{Y}_{d q}^{L_{2}} \mathbf{Z}_{d q}^{C}}{1+\left(\mathbf{Y}_{d q}^{L_{1}}+\mathbf{Y}_{d q}^{L_{2}}\right) \mathbf{Z}_{d q}^{C}} \\
\mathbf{Y}_{d q}^{o}=\frac{\left(1+\mathbf{Y}_{d q}^{L_{1}} \mathbf{Z}_{d q}^{C}\right) \mathbf{Y}_{d q}^{L_{2}}}{1+\left(\mathbf{Y}_{d q}^{L_{1}}+\mathbf{Y}_{d q}^{L_{2}}\right) \mathbf{Z}_{d q}^{C}}
\end{gathered}
$$

In (19), $\mathbf{Y}_{d q}^{\mathrm{cl}}$ is the admittance without considering the effects of the SRF-PLL and the ICFF, $\mathbf{Y}_{d q}^{\text {pllt }}$ is the admittance due the effect of the SRF-PLL on $\mathbf{i}_{d q}^{\text {ref }}$ ig and $\mathbf{v}_{d q}^{\mathrm{ref}}$, and $\mathbf{Y}_{d q}^{\mathrm{de}}$ is the admittance due the effect of the ICFF only.

The total admittance of the system, $\mathbf{Y}_{d q}^{t}$, is given by

$$
\mathbf{Y}_{d q}^{t}=\mathbf{Y}_{d q}^{\mathrm{cl}}+\mathbf{Y}_{d q}^{\mathrm{pllt}}+\mathbf{Y}_{d q}^{\mathrm{de}}
$$

that is equal to

$$
\mathbf{Y}_{d q}^{t}=\frac{\mathbf{Y}_{d q}^{o}-G_{d} \mathbf{Y}_{d q}^{p}\left(G_{c} \mathbf{Y}_{d q}^{\mathrm{pll}}+\mathbf{G}_{d q}^{\mathrm{pll}}\right)+\mathrm{j} \omega_{1} L G_{d} \mathbf{Y}_{d q}^{p} \mathbf{Y}_{d q}^{\mathrm{pll}}}{1+\left(G_{c}-\mathrm{j} \omega_{1} L\right) G_{d} \mathbf{Y}_{d q}^{p}}
$$

when ICFF is used and

$$
\mathbf{Y}_{d q}^{t}=\frac{\mathbf{Y}_{d q}^{o}-G_{d} \mathbf{Y}_{d q}^{p}\left(G_{c} \mathbf{Y}_{d q}^{\mathrm{pll}}+\mathbf{G}_{d q}^{\mathrm{pll}}\right)}{1+G_{c} G_{d} \mathbf{Y}_{d q}^{p}}
$$

when not.

Therefore, when ICFF is used, the total admittance of the system is modified, which results in a decrease in its stability margins.

\section{Vi. Generalized Nyquist Criterion}

According to the multivarible linear system theory [16], the stability of a MIMO system can be predicted based on the Generalized Nyquist Criterion (GNC). In simple terms, the GNC states that a MIMO system, L $(s)$, will be stable if the Nyquist plot of the determinant of $\mathrm{I}+\mathrm{L}(\mathrm{j} \omega)$ does not encircle the origin when $\omega$ changes from $-\infty$ to $+\infty$.

Applied to the system in Fig. 6, the GNC takes the form of

$$
\left|\mathrm{I}+\mathrm{Y}_{d q}^{t} \mathrm{Z}_{d q}^{g}(\mathrm{j} \omega)\right|
$$

where $\mathrm{Y}_{d q}^{t}$ and $\mathrm{Z}_{d q}^{g}$ are the $2 \times 2$ matrix representation of $\mathbf{Y}_{d q}^{t}$ and $\mathbf{Z}_{d q}^{g}$, and I is the identity matrix.

Fig. 7 shows a zoomed view of the Nyquist diagram of the system with and without ICFF for the 100kVA VSC, where it is observed that the additional admittance

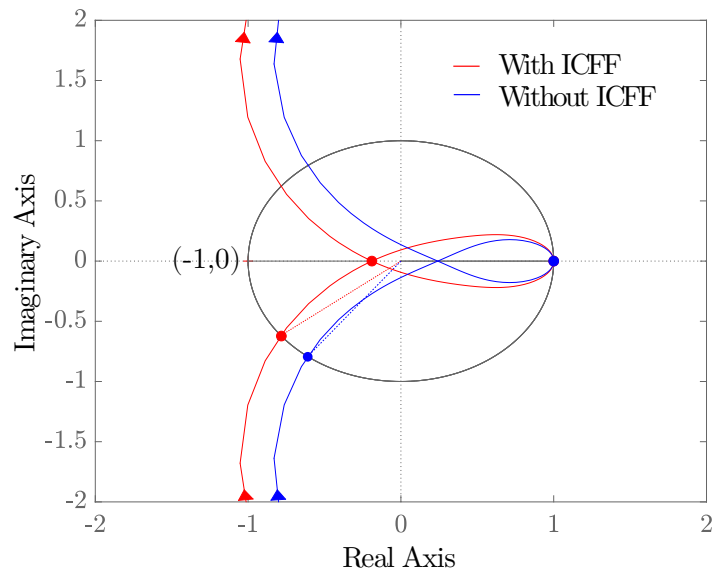

Fig. 7: Nyquist plots of $\left|\mathrm{I}+\mathrm{Y}_{d q}^{t} \mathrm{Z}_{d q}^{g}(\mathrm{j} \omega)\right|$ for the $100 \mathrm{kVA}$ VSC.

resulting from the decoupling term when ICFF is activated, $\mathrm{Y}_{d q}^{\mathrm{de}}$, decreases the stability margin of the system, therefore reducing the relative stability compared to when the ICFF is not activated. In the figure, the system is stable if the ICFF is not used and unstable if it used.

\section{Experimental Results}

Hardware-in-the-loop (HIL) simulations were conducted in order to validate the theoretical results obtained. The VSC was implemented on the Typhoon HIL platform model 600 (8/16 analog inputs/outputs). The control loop was discretized and carried out by means of the commercial DSP F28335 of Texas Instruments, where the calculation step is $1 \mu \mathrm{s}$.

The waveforms of the PCC voltage are shown in Fig. 8 . At the start of both cases, the controller operates without the ICFF component and the system response is seen to be stable. After a moment, the ICFF is activated and it is observed that the waveforms become significantly distorted, thus indicating a decrease in the stability margin of the system. The change is more noticeable for the 4 MVA VSC case, as shown in Fig. 8b.

\section{Conclusion}

This paper assesses the effect of using a decoupling technique on small-signal stability. First, a small-signal model is developed to observe the effect of the variation of parameters on the eigenvalues of the system under study. Then, the Nyquist diagrams based on the ac impedance are plotted to observe the reduction in the gainand phase-margin when the ICFF is activated. Thus, it is shown that changing parameters in a manner which would ordinarily improve the controller's performance, such as increasing the PLL bandwidth, in fact reduces the effectiveness of the controller when ICFF is activated. HIL experiments confirm the results obtained from the model and simulation. 


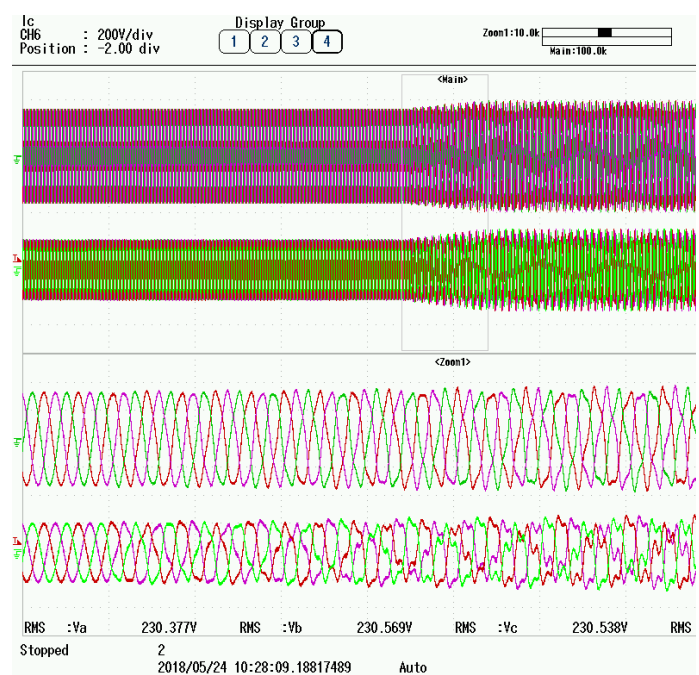

(a) $100 \mathrm{kVA}$.

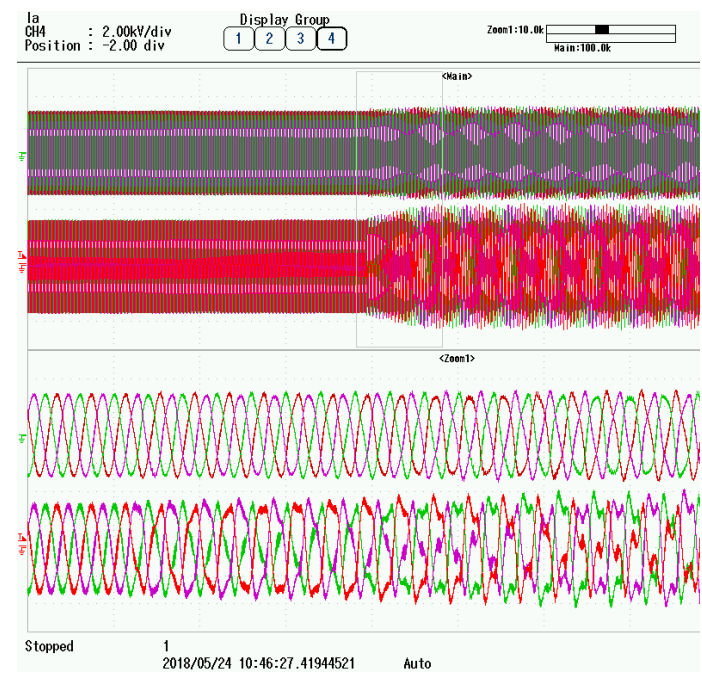

(b) 4 MVA.

Fig. 8: PCC voltage before and after activating the ICFF.

\section{ACKNOWLEDGMENT}

This work was supported by the European Commission under the project FLEXITRANSTORE - H2020-LCE-2016-2017-SGS-774407 and by the Spanish Ministry of Science Innovation and Universities under project SMARTNODES ENE2017-88889-C2-1-R. Any opinions, findings and conclusions or recommendations written in this work are those of the authors and do not necessarily reflect those of the host institutions and funders.

\section{REFERENCES}

[1] World Energy Council, World Energy Perspectives Variable Renewable Integration in Electricity Systems: How to get it right, 2016.
[2] B. Bahrani, S. Kenzelmann, and A. Rufer, "Multivariable PI-based $d q$ current control of voltage source converters with superior axis decoupling capability," IEEE Transactions on Industrial Electronics, vol. 58, no. 7, pp. 3016-3026, July 2011.

[3] S. Zhou, J. Liu, L. Zhou, and Y. Zhang, "DQ current control of voltage source converters with a decoupling method based on preprocessed reference current feed-forward," IEEE Transactions on Power Electronics, vol. 32, no. 11, pp. 8904-8921, Nov 2017.

[4] A. G. Yepes, A. Vidal, O. López, and J. Doval-Gandoy, "Evaluation of techniques for cross-coupling decoupling between orthogonal axes in double synchronous reference frame current control," IEEE Transactions on Industrial Electronics, vol. 61, no. 7, pp. 3527-3531, July 2014.

[5] F. Blaabjerg, R. Teodorescu, M. Liserre, and A. V. Timbus, "Overview of control and grid synchronization for distributed power generation systems," IEEE Transactions on Industrial Electronics, vol. 53, no. 5, pp. 1398-1409, Oct 2006.

[6] X. Wang, L. Harnefors, and F. Blaabjerg, "Unified impedance model of grid-connected voltage-source converters," IEEE Transactions on Power Electronics, vol. 33, no. 2, pp. 1775-1787, Feb 2018.

[7] P. Kundur, Power System Stability and Control, ser. The EPRI Power System Engineering Series. New York: McGraw-Hill, Inc., 1994.

[8] J. Sun, "Impedance-based stability criterion for grid-connected inverters," IEEE Transactions on Power Electronics, vol. 26, no. 11, pp. 3075-3078, Nov 2011.

[9] X. Wang, F. Blaabjerg, and W. Wu, "Modeling and analysis of harmonic stability in an ac power-electronics-based power system," IEEE Transactions on Power Electronics, vol. 29, no. 12, pp. 6421-6432, Dec 2014.

[10] B. Wen, D. Boroyevich, R. Burgos, P. Mattavelli, and Z. Shen, "Analysis of d-q small-signal impedance of grid-tied inverters," IEEE Transactions on Power Electronics, vol. 31, no. 1, pp. 675-687, Jan 2016.

[11] L. Harnefors, M. Bongiorno, and S. Lundberg, "Input-admittance calculation and shaping for controlled voltage-source converters," IEEE Transactions on Industrial Electronics, vol. 54, no. 6, pp. 3323-3334, Dec 2007.

[12] D. Pan, X. Ruan, C. Bao, W. Li, and X. Wang, "Capacitor-current-feedback active damping with reduced computation delay for improving robustness of LCL-type grid-connected inverter," IEEE Transactions on Power Electronics, vol. 29, no. 7, pp. 3414-3427, Jul 2014.

[13] L. Harnefors, A. G. Yepes, A. Vidal, and J. Doval-Gandoy, "Passivity-based stabilization 
of resonant current controllers with consideration of time delay," IEEE Transactions on Power Electronics, vol. 29, no. 12, pp. 6260-6263, Dec 2014.

[14] K. Sharifabadi, L. Harnefors, H. Nee, S. Norrga, and R. Teodorescu, Design, control, and application of modular multilevel converters for HVDC transmission systems. John Wiley \& Sons, 2016.

[15] L. Marin, A. Tarraso, I. Candela, and P. Rodriguez, "Stability analysis of a droop-controlled grid-connected VSC," in 2018 IEEE Energy Conversion Congress and Exposition (ECCE), Sep. 2018, pp. 4161-4167.

[16] S. Skogestad and I. Postlethwaite, Multivariable feedback control: analysis and design, 2nd ed. Hoboken, NJ: John Wiley \& Sons, 2005. 